\title{
Essential Elements of and Challenges to Rapid ART Implementation: A Qualitative Study of Three Programs in the United States
}

Kimberly A Koester ( $\nabla$ Kimberly.koester@ucsf.edu )

University of California, San Francisco

Lissa Moran

University of California, San Francisco

Noelle LeTourneau

University of California, San Francisco

Lyndon VanderZanden

Howard Brown Health Center

Susa Coffey

University of California, San Francisco

Pierre-Cedric Crouch

San Francisco AIDS Foundation

Janessa Broussard

San Francisco AIDS Foundation

John Schneider

Howard Brown Health Center

Katerina A. Christopoulos

University of California, San Francisco

\section{Research Article}

Keywords: HIV treatment, Rapid ART, Immediate ART Qualitative Methods

Posted Date: November 10th, 2021

DOl: https://doi.org/10.21203/rs.3.rs-966088/v1

License: (c) (i) This work is licensed under a Creative Commons Attribution 4.0 International License. Read Full License

Version of Record: A version of this preprint was published at BMC Infectious Diseases on March 31st, 2022. See the published version at https://doi.org/10.1186/s12879-022-07297-3. 


\section{Abstract}

Background: Antiretroviral therapy (ART) initiation on the day of an HIV diagnosis or as soon as possible after diagnosis, known as rapid ART (henceforth "RAPID"), is considered to be a safe and effective intervention to quickly reduce viral load and potentially improve engagement in care over time. However, implementation of RAPID programming is not yet widespread. To facilitate broader dissemination of RAPID, we sought to understand health care worker experiences with RAPID implementation and to identify essential programmatic elements.

Methods: We conducted 28 key informant interviews with medical providers and staff involved in RAPID service delivery in three distinct clinical settings. Interviews were audio-recorded, transcribed, and thematically analyzed.

Findings: We identified seven (7) essential elements across settings associated with successful RAPID program implementation. These high-impact elements represent essential components without which a RAPID program could not function. There was no one requisite formation. Instead, we observed a constellation of essential elements that could be operationalized in various formations and by various people in various roles. The essential elements included: (1) presence of an implementation champion; (2) comfort and competence prescribing RAPID ART; (3) expedited access to ART medications; (4) expertise in benefits, linkage, and care navigation; (5) RAPID team member flexibility and organizations' adaptive capacity; (6) patient-centered approach; and (7) strong communication methods and culture.

Conclusions: The RAPID model can be applied to a diverse range of clinical contexts. The operational structure of RAPID programs is shaped by the clinical setting in which they function, and therefore the essential elements identified may not apply equally to all programs. Based on the seven essential elements described above we recommend future implementers identify where these elements currently exist within a practice; leverage them when possible; strengthen them when necessary or develop them if they do not yet exist; and look to these elements when challenges arise for potential solutions.

\section{Background}

Advances in the treatment of HIV have evolved substantially over the last three decades. Consistent use of antiretroviral therapy (ART) allows individuals to achieve and maintain optimal health, while rates of onward HIV transmission are virtually eliminated in the when viral load is durably suppressed. ${ }^{1,2}$ Healthcare delivery systems must similarly evolve to fully realize the benefits of HIV treatment advances. This includes identifying effective strategies to meaningfully link and retain individuals in care in the immediate and longer term.

Early initiation of ART confers clinical and public health benefits. ${ }^{3-8}$ ART initiation on the day of diagnosis or as soon as possible after diagnosis, known as rapid or immediate ART (henceforth "RAPID"), is considered to be a safe and effective intervention to quickly reduce viral load and potentially improve engagement in care over time. ${ }^{9-11}$ Yet the implementation of RAPID programming is not yet widespread. 
This may be due, in part, to evidence suggesting that implementation of RAPID programming is resourceintensive. $^{12-14}$ Just how resource intensive is an open question, and key questions about optimal implementation of RAPID remain.

Researchers have called for studies assessing how RAPID (as a collection of immediate linkage and medication initiation services) is operationalized in different U.S. healthcare settings. ${ }^{15,16}$ Understanding how distinct clinical settings operationalize the component parts of RAPID-i.e., immediate access to medical and psychosocial assessment, assistance with establishing insurance benefits, intensive HIV counseling and education, ordering of baseline laboratory tests, provision of ART medications and linkage to HIV primary care services-could be critical to the widespread scale up of programs. ${ }^{13,14,17}$ Providing an assessment of the types of implementation strategies that accelerate access to ART and retention in care will also move the field forward.

To that end, we conducted multi-site qualitative research to assess RAPID program implementation efforts from the perspective of providers and staff. Our work provides evidence on the necessary resources and systems-level transformations needed to adopt the RAPID model in a variety of clinical settings. The purpose of this article is to outline the essential elements and challenges of RAPID implementation across three distinct settings.

\section{Methods}

\section{Study Design and Settings}

This paper reports on a series of studies unified by a focus on assessing RAPID implementation processes. Initially, we designed a qualitative study to understand provider/staff perspectives on RAPID implementation in the San Francisco-based HIV clinic that first pioneered RAPID in the United States. ${ }^{15}$ We subsequently added two sites in order to study adaptations to the original model. Table 1 depicts the organizational characteristics of each setting. Briefly, the HIV clinic (henceforth Innovator) began implementing RAPID ART in 2013. Inspired by the success of the original RAPID model, a federally qualified health center (henceforth FQHC) in Chicago launched their own program based on the San Francisco model in 2018. Also in 2018, a robust HIV testing program situated within a sexual health and wellness clinic (henceforth Testing Site) in San Francisco launched its own RAPID program. As a setting that did not provide primary care, this particular RAPID model was distinct in that they started clients on ART and then linked them to an HIV care provider for ongoing care.

Table 1: Study Site Characteristics 


\begin{tabular}{|c|c|c|c|}
\hline $\begin{array}{l}\text { Site } \\
\text { characteristics }\end{array}$ & Innovator Site & FQHC & Testing Site \\
\hline Clinic Type & $\begin{array}{l}\text { Academic HIV clinic } \\
\text { in safety-net hospital } \\
\text { system }\end{array}$ & Federally Qualified Health Center & $\begin{array}{l}\text { Sexual health } \\
\text { clinic and testing } \\
\text { site }\end{array}$ \\
\hline $\begin{array}{l}\text { RAPID patient } \\
\text { source }\end{array}$ & $\begin{array}{l}\text { Combination of } \\
\text { patients diagnosed } \\
\text { w/in hospital system } \\
\text { \& off site }\end{array}$ & $\begin{array}{l}\text { Combination of patients diagnosed on } \\
\text { and off site }\end{array}$ & $\begin{array}{l}\text { Majority of } \\
\text { patients } \\
\text { diagnosed on } \\
\text { site }\end{array}$ \\
\hline $\begin{array}{l}\text { RAPID visit } \\
\text { structure }\end{array}$ & $\begin{array}{l}\text { By appointment \& } \\
\text { drop-in }\end{array}$ & By appointment \& drop-in & $\begin{array}{l}\text { By appointment } \\
\text { \& drop-in }\end{array}$ \\
\hline RAPID Team & $\begin{array}{l}\text { Prescriber (MD, NP) } \\
+\mathrm{RN}+\mathrm{MSW}\end{array}$ & $\begin{array}{l}\text { Health Educator (HE), RN or MA + } \\
\text { Prescriber (MD, DO, NP, PA, APN) }+ \\
\text { Linkage to Care Coordinator (LTC) }+ \\
\text { Pharmacist }\end{array}$ & $\begin{array}{l}\text { Prescriber (NP) + } \\
\text { Health Navigator }\end{array}$ \\
\hline $\begin{array}{l}\text { Composition } \\
\text { of RAPID Visit }\end{array}$ & $\begin{array}{l}\text { Typically, patient } \\
\text { seen by whole } \\
\text { RAPID team at once }\end{array}$ & $\begin{array}{l}\text { Typically, patient seen by RN, HE, or } \\
\text { MA, then LTC, then Prescriber }\end{array}$ & $\begin{array}{l}\text { Typically, patient } \\
\text { seen by Health } \\
\text { Navigator and } \\
\text { NP only }\end{array}$ \\
\hline $\begin{array}{l}\text { Linkage to HIV } \\
\text { Primary Care }\end{array}$ & $\begin{array}{l}\text { HIV primary care on- } \\
\text { site (no external } \\
\text { linkage) }\end{array}$ & $\begin{array}{l}\text { Within FQHC system (linkage to } \\
\text { internal primary care at one of } 10 \\
\text { clinics) }\end{array}$ & $\begin{array}{l}\text { Off-site } \\
\text { (linkage to } \\
\text { external primary } \\
\text { care) }\end{array}$ \\
\hline $\begin{array}{l}\text { \# of RAPID } \\
2019 \\
\text { Encounters }\end{array}$ & 41 & 198 & 65 \\
\hline
\end{tabular}

\section{Sampling, Recruitment and Data Collection Procedures}

At each site, providers and staff who ran or oversaw the program facilitated identification of prospective key informants. To be eligible for the study, providers and staff had to be involved in RAPID service delivery. Authors KK or LM reached out to potential key informants via email to invite them to join the study, using a standard script and offering to schedule an interview at a mutually convenient time. Depending on logistics, such as the availability of a private space to conduct an interview and the location of the key informant, interviews at the sites in San Francisco were either conduced over the phone (2) or in-person (19). All interviews conducted with FQHC key informants were conducted over the phone due to the geographic distance between the San Francisco-based research team and the FQHC site. Both KK and LM are qualitative researchers with extensive experience building rapport and conducting in-depth interviews with HIV providers/staff. KK and KAC developed the interview guide with domains associated with the Consolidated Framework for Implementation Research (CFIR) in mind: intervention characteristics, inner and outer settings, individual characteristics, and implementation 
process. ${ }^{18}$ While our findings are not presented in alignment with CFIR domains and constructs, the framework's multi-level and -dimensional implementation considerations defined the scope of the interview guide and thus the universe of the data we collected. Interview domains included preimplementation and implementation experiences, attitudes about RAPID, and patient experiences. Interviews lasted up to 60 minutes, were audio-recorded, and transcribed verbatim. Each key informant was offered a $\$ 20$ incentive as a token of appreciation.

\section{Data Analysis}

After each interview, KK or LM drafted a fieldnote to capture a synopsis of the interview content, changes to the interview guide, emerging themes, and any additional information related to the interview that would not be captured in the transcript. These fieldnotes were distributed to the broader team and discussed during meetings. Initially, our descriptive analysis consisted of a deductive examination of the implementation experiences at the Innovator Site. Authors KK and LM reviewed the fieldnotes and transcripts associated with all the interviews from that site and drafted analytic memos based on each interview. These memos were compared across informants and an overall memo depicting key themes related to the implementation process, essential elements and challenges was developed for the Innovator Site. This summary informed modifications to the guide used during interviews with key informants in the Testing and FQHC sites. It was eventually used as a foundation to compare and contrast interview data from each of the other RAPID programs.

As we accumulated more data, we modified our analytic strategy to include coding. We also integrated an additional person to our analytic team (NLT). NLT was the data manager for the study and had prior experience with qualitative research; her familiarity with the quantitative data being collected for the study offered a unique perspective to the analytic team. The three analysts collectively developed a codebook following a process authors KK and LM have successfully used in the past. This process included reading a transcript selected for its data richness aloud to discuss, interpret and collectively generate a list of preliminary codes. Once a preliminary list of codes was drafted, we independently coded 2 additional interviews and met to cross-check our coding choices. We further refined the codebook by elaborating on meanings and dropping or adding codes. The remaining interviews were divided across the team, with each transcript assigned to a primary coder and a secondary reviewer. Once the data were coded, verified and entered into Dedoose ${ }^{19}$, the team developed a data reduction plan. Our chief goal during the analysis process was to compare and contrast across cases within a site and then to expand comparison across sites. This allowed us to identify similarities, differences and deviant or negative cases. We generated code reports within Dedoose for 13 codes (codebook available upon request) to read and summarize. During bi-weekly data reduction sessions, we documented our collective interpretations and analytic insights. These data summaries were the basis for our identification of essential elements and implementation challenges presented below. We held a study report back session (member check) to share our preliminary findings with a subset of our key informants. ${ }^{20}$ That session sensitized our team to the findings that were thought to be maximally useful to the field and led to some minor but important adjustments in the representation of some aspects of the programs. 


\section{Findings}

We conducted 27 interviews from March 2018 to February 2020 (see Table 2 for participant demographics). We identified seven (7) essential elements across settings associated with successful RAPID program implementation. We provide examples of the application of these essential elements and indicate if and how they functioned differently in each setting (see Table 3). We also highlight ongoing operational challenges to inform future implementation efforts elsewhere.

Table 2. Participant Demographics

\begin{tabular}{|ll|}
\hline $\mathrm{N}=27$ & $\mathrm{~N}(\%)$ \\
\hline Gender & \\
\hline Male & $10(37 \%)$ \\
\hline Female & $15(56 \%)$ \\
\hline Trans/Non-Binary & $2(7 \%)$ \\
\hline Race/Ethnicity & \\
\hline White & $19(70 \%)$ \\
\hline Latinx & $6(22 \%)$ \\
\hline Asian & $1(4 \%)$ \\
\hline Black & $1(4 \%)$ \\
\hline Years in HIV & \\
\hline$<5$ & $9(33 \%)$ \\
\hline 5 to 15 & $10(37 \%)$ \\
\hline$>15$ & $8(30 \%)$ \\
\hline Role within RAPID program & $11(4 \%)$ \\
\hline Prescribing provider (MD, NP, PharmD) & $11(41 \%)$ \\
\hline RN & $4(15 \%)$ \\
\hline Social work/Navigator/Linkage Specialist & $11(41 \%)$ \\
\hline Leadership & $1(4 \%)$ \\
\hline
\end{tabular}

Table 3. Essential elements of RAPID, operationalized by site 


\begin{tabular}{|c|c|c|c|}
\hline & Innovator Site & Testing Site & FQHC \\
\hline \multirow[t]{3}{*}{$\begin{array}{l}\text { Comfort and } \\
\text { competence } \\
\text { prescribing ART }\end{array}$} & $\begin{array}{l}\text { MDs/NPs } \\
\text { prescribing }\end{array}$ & $\begin{array}{l}\text { NPs prescribing (recent } \\
\text { addition for other prescribing } \\
\text { services at site) }\end{array}$ & $\begin{array}{l}\text { MDs/NPs/DOs/PAs/APNs } \\
\text { prescribing }\end{array}$ \\
\hline & $\begin{array}{l}\text { HIV primary } \\
\text { care team w } \\
\text { expertise in HIV } \\
\text { care }\end{array}$ & $\begin{array}{l}\text { All providers available to } \\
\text { participate in RAPID as any } \\
\text { other sexual health service }\end{array}$ & $\begin{array}{l}\text { HIV primary care team w } \\
\text { expertise in HIV care }\end{array}$ \\
\hline & $\begin{array}{l}\text { Select cadre of } \\
\text { RAPID providers } \\
\text { from broader } \\
\text { pool of primary } \\
\text { care providers }\end{array}$ & & $\begin{array}{l}\text { All providers available to } \\
\text { participate in RAPID as any } \\
\text { other sexual health service }\end{array}$ \\
\hline \multirow[t]{3}{*}{$\begin{array}{l}\text { Expedited } \\
\text { access to ART }\end{array}$} & $\begin{array}{l}\text { Starter packs + } \\
\text { Rx for ongoing } \\
\text { ART to be filled }\end{array}$ & Starter packs + Rx & $\begin{array}{l}\text { Prescription that could be } \\
\text { filled immediately or next- } \\
\text { day at the on-site pharmacy }\end{array}$ \\
\hline & & $\begin{array}{l}\text { Proximity to pharmacy for } \\
\text { immediate prescription } \\
\text { fulfillment (same-day Rx } \\
\text { fulfillment so successful, } \\
\text { starter packs rendered } \\
\text { unnecessary) }\end{array}$ & $\begin{array}{l}\text { Proximity to pharmacy for } \\
\text { immediate prescription } \\
\text { fulfillment }\end{array}$ \\
\hline & & & Medication sample packs \\
\hline \multirow[t]{4}{*}{$\begin{array}{l}\text { Benefits, } \\
\text { linkage, and } \\
\text { care navigation }\end{array}$} & $\begin{array}{l}\text { Access to } \\
\text { patient drug } \\
\text { assistance } \\
\text { programs (same } \\
\text { day ADAP) }\end{array}$ & $\begin{array}{l}\text { Access to patient drug } \\
\text { assistance programs (ADAP) }\end{array}$ & $\begin{array}{l}\text { Access to patient drug } \\
\text { assistance programs } \\
\text { (ADAP) }\end{array}$ \\
\hline & $\begin{array}{l}\text { Clinic receives } \\
\text { warm hand-offs } \\
\text { from referrals: }\end{array}$ & $\begin{array}{l}\text { Ability to assess eligibility and } \\
\text { enroll patients in benefits } \\
\text { programs same-day }\end{array}$ & $\begin{array}{l}\text { Insurance and patient } \\
\text { assistance program } \\
\text { knowledge for patients to } \\
\text { access free meds }\end{array}$ \\
\hline & $\begin{array}{l}\text { on-site HIV } \\
\text { primary care, no } \\
\text { external linkage } \\
\text { necessary }\end{array}$ & $\begin{array}{l}\text { Assess for additional needs } \\
\text { (housing, mental health, etc.), } \\
\text { psychosocial support on site }\end{array}$ & $\begin{array}{l}\text { Clinic receives referrals as } \\
\text { warm hand-offs and self- } \\
\text { referrals; on-site HIV } \\
\text { primary care, no external }\end{array}$ \\
\hline & $\begin{array}{l}\text { Social worker as } \\
\text { part of RAPID } \\
\text { team to assess } \\
\text { and connect pt } \\
\text { to wraparound } \\
\text { services }\end{array}$ & $\begin{array}{l}\text { Off-site linkage to external HIV } \\
\text { PC provider (includes } \\
\text { assessments of benefits and } \\
\text { needs to find appropriate } \\
\text { match, coordinating } \\
\text { transition) }\end{array}$ & $\begin{array}{l}\text { Assess for additional needs } \\
\text { (housing, mental health, } \\
\text { etc.), psychosocial support } \\
\text { on site }\end{array}$ \\
\hline
\end{tabular}


Navigation of pt

through RAPID

process ("red

carpet"

treatment)
Navigation of pt through RAPID process, ensuring warm hand-off to internal HIV PC provider
Flexibility and adaptive capacity

\section{Appointment \& Drop-in}

Interdisciplinary

Team

Prescribing

clinicians

flexible in

accommodating

RAPIDs in the

schedule
Appointment \& Drop-in

Interdisciplinary Team

Iterative program development and improvement (i.e., crosstraining benefits navigators and health navigators; expanding physical space for RAPID; opening access to mental health services at RAPID visit when needed)
Appointment \& Drop-in

Interdisciplinary Team

Iterative program development and improvement (i.e., adding Uber account, adding onsite linkage coordinator to clinic)

Iterative

program

development

and

improvement

\section{Patient-} centered approach
Services for patients experiencing homelessness, substance use

RAPID as a redcarpet event to provide patient support

Warm hand offs between RAPID team members
LGBTQ+ community focus, including transgender and gender nonbinary services

Community outreach and sitespecific access (for patients experiencing homelessness), mobile testing units

Meet with as few people as possible during encounter

Attitude of doing whatever it takes to meet the patient's need
LGBTQ+ community focus, including transgender and gender nonbinary services

Services for patients experiencing homelessness, mental health needs, substance use

Community outreach and testing

Meet with as few people as possible during encounter, stays in same exam room throughout process, (when possible/desirable) has prescription brought to them 
Attitude of doing whatever it takes to meet the patient's need

Accessing all needs for patients (i.e., clothing, food, transportation vouchers)

In-house linkage to minimize time patient spends alone

Communication methods and culture
Interdisciplinary team, all team members present and together for RAPID encounter

Central pager system
Small team communicates informally throughout daily activities
Centralized "linkage to care phone"

Repeated check-ins among RAPID team throughout RAPID process, and continued in-person and EMR-based communication throughout full linkage to care process (first 6-12 months of patient's care)

RAPID team members use EMR to read patient notes during RAPID encounter

\section{Essential Elements for RAPID Program Implementation}

Key informants from each site detailed barriers and facilitators to successful implementation of RAPID. From these, seven high-impact elements emerged that represent essential components without which a RAPID program could not function. There was no one requisite formation. Instead, we observed a constellation of essential elements that could be operationalized in various formations and by various people in various roles. The essential elements included: (1) presence of an implementation champion; (2) comfort and competence prescribing RAPID ART; (3) expedited access to ART medications; (4) expertise in benefits, linkage, and care navigation; (5) RAPID team member flexibility and organizations' adaptive capacity; (6) patient-centered approach; and (7) strong communication methods and culture.

\section{(1) Implementation champion}


The health services implementation science literature supports the importance of a champion in quality improvement and implementation efforts. ${ }^{21-23}$ Among the sites in this study, each had one or more individuals who served as a champion invested in the success of the RAPID intervention. In the case of the Innovator Site, the champions were in positions of leadership and instructed key staff to offer patients ART on the day of diagnosis. One interviewee recollected thinking that prior to embarking upon RAPID program implementation, it would be "impossible" to have a patient start on medications on the day of disclosure. She then explained how quickly that "impossible" mindset changed:

"Okay. We'll try it," but we weren't very confident that it could work. . we were completely convinced, within three or four days. The lesson was it was an unbelievably empowering thing for the patients. It stopped them as they were going down the rabbit hole of HIV stigma that comes with a new diagnosis. It just could stop that process and really make people feel like, at the end of their visit - that they were walking out with a sense of hope and that this was a manageable, chronic condition." (Innov KIO1)

The champions in the Testing and FQHC sites were motivated by the success of the Innovator Site and were eager to have patients realize the benefits of RAPID ART. Collectively, RAPID champions drove the implementation process forward and were instrumental in overcoming challenges that might have otherwise derailed successful implementation.

(2) Prescribing: at least one individual on the team possesses comfort and competence in prescribing same-day ART

As a medical intervention, a RAPID program requires a provider with prescribing privileges to initiate immediate ART for newly diagnosed patients on an as-needed basis, and a willingness to do so. Key informants from the sites that provide primary care (Innovator and FQHC) indicated that some prescribers were well-suited to conducting a RAPID visit because they felt comfortable prescribing ART in the absence of initial laboratory results and because they were amenable to the disruption of drop-in visits. Within the Testing Site, implementing RAPID was made possible because they had recently expanded their workforce, adding nurse practitioners (NPs) to provide hepatitis $\mathrm{C}$ treatment and HIV pre-exposure prophylaxis (PrEP) as new services. Prior to that, they did not have staff with prescribing privileges in their nurse-led clinic.

Finding providers with knowledge of HIV treatment was not an issue at any of the sites participating in this study, but given the national shortage of HIV providers, it could be a problem in other clinic settings. ${ }^{24,25}$ In addition to basic competencies in HIV care, RAPID requires provider acceptability-belief that the model is beneficial for patients, that it will not do patients harm, and that the value of immediate ART outweighs the risks of starting ART before initial laboratory (i.e., genotype, HIV RNA, CD4 and renal function) results are available.

"I'd rather err on the side - 'cause worst case scenario, you get them the pills, they take one, and then you never see them again-they don't take anymore. And like, they're not going to cause themselves that much harm from it. I would be horrified, horrified if I did not give a treatment to somebody who would've 
successfully done it. I'd rather ... err, whatever else, giv[e] someone the opportunity who maybe wasn't ready than to remotely risk someone not getting care." (Test KI04)

The quote above illustrates this prescriber's risk-benefit calculation. Notably they expressed confidence in RAPID ART as an intervention to facilitate retention in care. Key to the success of RAPID programming is identifying at least one provider with the ability to prescribe medication, who has the comfort and confidence in the RAPID model and in their own knowledge of HIV care and treatment to prescribe ART at the time of diagnosis.

\section{(3) Expedited access to ART medications.}

Accessing ART within an expedited timeframe was identified as a discrete competency, one that involved expertise in insurance eligibility assessment, payer systems, and funding mechanisms, such as same-day ADAP enrollment or pharmaceutical programs that offer copay cards. Our Innovator Site, for example, relied on "starter packs" of ART (purchased by the clinic) in addition to a prescription, so all patients could start ART while ADAP and Medicaid eligibility was established. The FQHC site took a similar approach, but utilized pharmacy access programs and pharmaceutical samples, rather than starter packs. RAPID patients at the FQHC site were provided a prescription for immediate or next-day fill at an onsite or local pharmacy, and if necessary, were enrolled in a pharmacy access program or given pharmaceutical samples if they would have otherwise had to delay initiation until ADAP and Medicaid enrollment was completed. The Testing Site had multiple access points to expedited access to ART medications, such as starter packs and same-day pick-up at nearby pharmacies, which could be tailored to a patient's need and around a variety of logistical or administrative constraints.

"They leave with five days of meds. So, it's not just like, "Okay, here's this prescription," because there's still 1,000 things that can go wrong between, like, handing them a prescription and picking up that prescription from your, you know, local, unfriendly Walgreens, and so ... Yeah, starter packs are amazing." (Innov K104)

As a high-volume PrEP provider, the Testing Site had a lot of experience with and clear processes in place to facilitate efficient access to PrEP medication. Staff transferred these skills over to facilitate expedited access to ART.

(4) Benefits, linkage, and care navigation.

Benefits, linkage, and care navigation represent a suite of essential skill areas for RAPID operation. Benefits navigation encompasses all activities associated with eligibility assessments and enrollments into benefits, including health insurance, drug assistance programs like ADAP, and access to support programs. RAPID support staff who can quickly and competently navigate insurance and funding mechanisms provide critical support to patients. A Testing Site informant explained the crucial role played by a "dedicated benefits team": 
"Having the nurses, well trained in sexual health and HIV treatment is definitely one of the most [important] things that made this program successful, and also having a dedicated benefits team. Without that you can have the best nurse that's well versed in all aspects of HIV treatment, but if they're not well versed in how to get the patient the medications.... It's just gonna end right there. Yes, you have great care, but can you afford your medication when you leave the clinic? No." (Test K109)

Same-day access to insurance and benefits programs provide the material benefits of RAPID ART and ongoing HIV care services for low- or no-cost to patients, as well as psychosocial support to patients for whom concerns about paying for care and treatment are a barrier. An FQHC interviewee spoke about the importance of communicating about insurance options to the patient early on to reduce anxiety and/or misperceptions related to the cost of HIV care and treatment.

"... the number one question I usually get from people when I talk to them, is how much is this all going to cost me? So being able to say confidently that we have the resources to get you all of this for free, I think is a big relief for patients, and a big relief in why they're able to initiate that." (FQHC KIO5)

Care navigation, such as identifying needed resources and making services available, represents the broader services in place to meet a patient's needs during a RAPID visit and can include emotional as well as material support. Across the sites, care navigation was performed by multiple service providers with different titles, among them patient or health navigator, social worker, nurse, linkage coordinator, and case manager. The person or persons in the care navigation role typically oversaw and facilitated a patient's movement through the RAPID process, assessing additional needs (housing, mental health, etc.), providing psychosocial support, and either physically escorting the patient from one provider station to another (exam room, benefits counselling, lab, pharmacy) or serving as the patient's single point of contact while other services were completed or procured behind the scenes.

Ideally, individuals who are diagnosed in community settings e.g., community-based HIV testing sites, sexual health clinics or emergency departments, rather than within an HIV care facility are provided linkage-to-care support. For example, an individual may be instructed to schedule an appointment right away at a local clinic and they may be provided with a list of local HIV care providers or in other cases, an appointment may be made on behalf of the individual or an individual may be provided with a warm hand-off and be personally escorted to an HIV care facility. In the case of our FQHC and Testing Sites, implementation of RAPID allowed a patient to initiate ART first and then be linked -within the same health center or externally-to ongoing primary care. Implementing RAPID allowed staff in these sites to slow down the linkage process, thus creating the opportunity to get to know a patient and make a more intentional linkage to care connection. One key informant from the Testing Site talked about how removing the urgency to get an individual quickly linked to a primary HIV care site allowed the health navigator to re-direct their attention to, among other things, tending to the emotional state of the client. Test_KI03 explained:

"Sitting with the client to me is one of the most important things, and establishing some rapport so that they can become increasingly comfortable to work with me and share some information that helps me 
figure out what's an appropriate medical home for them.... So having someone start on RAPID is invaluable because it gives us that breathing room. We don't have to, like, oh, my God, oh, my God, oh, my God, they have to start on medication, they have to, you know, get into care yesterday. But they're still sitting there shaking. We haven't even started the intake process, let alone, you know they don't have medical insurance and, you know, just all these variables. So it's just invaluable." (Test KI03)

(5) RAPID team member flexibility and organizations' adaptive capacity.

Providing RAPID services was, to varying degrees, disruptive in each setting. Success in these clinics was due in large part to each site having sufficient adaptive capacity. While there was no way to predict when a reactive HIV test would occur, all sites planned for RAPID service-related disruptions. Each site demonstrated flexibility and adaptive capacity differently; however, they shared two key components: each included capacity for drop-in visits; and each site engaged in an iterative process of feedback and correction to shape, modify, and hone their programs.

The drop-in element, and a clinic flow process built to absorb a certain amount of unpredictability, were crucial. The clinic scheduling system did not need to be primarily drop-in, but having a drop-in component at minimum-i.e., drop-in hours or appointment slots reserved for drop-ins-was essential for the flexibility to take on unpredictable appointments.

"I'd say more than half of our appointments are drop-in. It's like an open-access model, and that's where you get to squeeze in, you know, PrEPs, the PEPs [Post-exposure prophylaxis], the iARTs [ART reengagement], the RAPIDs." (Test KI08)

Many informants described a similarly flexible and adaptive approach to RAPID implementation and quality improvement. While formal pre-implementation activities such as engaging stakeholders and identifying barriers are considered best practice for implementing interventions, two sites implemented RAPID programs without engaging in such activities. The remaining site spent considerable time planning for and identifying potential barriers. They predicted that more linkage staff might be needed, however, confirmation of this prediction would need to be made after the program was initiated. Furthermore, no informants described personal involvement in a formal RAPID-related quality improvement protocol once implementation began. Many instead described RAPID program improvement resulting from an informal, ongoing process of feedback and correction. As a result, RAPID as a program was built and honed as it was in operation, and the iterative feedback loop of assessment and correction served as the primary mechanism of program development. In other words, as explained by an interviewee: "we really learned by doing it."

This process of iterative program development provided a channel for staff and providers to circulate feedback on what they needed individually to sustain their work-such as adding linkage staff once data on demand for RAPID services became clear to leadership in the FQHC site, or expanding physical space to accommodate an increased volume of RAPID patients at the Testing Site-and what they perceived to 
be the needs of the program in order for it to be better, more efficient, and sustainable in changing conditions.

"We're building out staffing to be able to see more folks as they drop in-so having more nurse practitioners. [A/so] we're expanding to the third floor to use one of the counseling rooms. I think we've already started using one of the counseling rooms up here for an additional like blood draw station. We're renovating our clinic to add another exam room on the second floor. So, there are like staffing and space needs that are being addressed to ideally build out that capacity." (Test KI11)

This process also provided a framework in which those performing RAPID services could identify and address ways to improve the patient experience as observed from their close patient contact-e.g., creating access to rideshare transportation services to ensure patients had a reliable and safe transportation option to attend HIV-related appointments.

(6) Patient-centered approach.

Implementation of RAPID programming in each site was a patient-centered endeavor. Not only was the well-being of the patient the primary motivation for implementing RAPID, but the content of each encounter was shaped around meeting the unique needs of each patient. For example, while providers laid out the rationale for starting ART, ultimately the decision rested with the patient, as an interviewee from the Innovator Site explained:

"I think with managing any kind of chronic illness, you can be nervous for your patient. You're just hopeful that they're able to follow directions or keep track of their meds or keep coming to their scheduled appointments. So, for me, yes, I definitely had reservations, and you still move forward and try and support people the best you can. Because if they say they want to start meds, it would feel so inappropriate to deny them medication... I think that it's important to just respect what a patient wants after they're given non-biased, nonjudgmental information and support. They get to decide the next step. And then you help create a care plan that makes it as easy as possible." (Innov KI05)

Even provider buy-in was patient-centered; some providers who initially harbored doubts about the sameday treatment model were convinced of the value of offering immediate ART once they saw the positive effect RAPID ART had on patients.

"Are we pressuring patients to do this? How is it really going to help them? I think fairly quickly we kind of got that from the patients... they really saw that as an opportunity to like take control over this virus that they thought was like out of control in their body. So, it was actually, actually pleasantly surprising. It was really a nice surprise because I was kind of worried." (Innov KIO3)

Common among the sites was the patient-centered goal of providing the patient with an interdisciplinary experience, even when RAPID services were not executed by an interdisciplinary team. 
"One of the very special things about this clinic but definitely the ... RAPID team is how interdisciplinary it is and how none of it would work if anybody was missing. Like, that there needs to be a nurse, and there needs to be social workers, and there needs to be eligibility, and there needs to be providers, and, like, it's such a beautiful example of the power of interdisciplinary work." (Innov KIO4)

Participants from the Innovator Site talked about the power of metaphoric handholding and creating a "red carpet" experience for a RAPID patient. RAPID team members at all sites strategically guided patients through all aspects of the visit, including minimizing unescorted time and providing warm hand-offs between providers. When done well, warm hand-offs can exemplify respect and trust between team members. Being around a dynamic of friendliness and comradery among RAPID team members could make a patient feel more comfortable with the various clinic staff to whom they were being handed off.

At the Innovator Site, the goal was to concurrently meet with the patient as a team to execute the full range of RAPID activities. At the Testing Site, one goal was to reduce the number of team members interacting with the patient in order to minimize the number of times the patient would have to disclose their new HIV status, ideally just a nurse practitioner and health navigator; other RAPID activities, including medication procurement, benefits eligibility assessment and lab processing, were executed by staff behind the scenes. At the FQHC the RAPID process and order of operations was flexible and depended on what would make for the most smooth and efficient process for the patient:

"So, instead of somebody finding out their diagnosis and me meeting with them right away, they'll meet with a provider first, and then they'll meet with like a [partner] service person, and then they'll meet with me. So, it's just the structure is a little bit different depending on like which clinic the person is diagnosed at." (FQHC KIO3)

When possible, the RAPID protocol at the FQHC was adjusted to meet patient needs and per the feedback of staff who were on the ground observing where, when, and how clinic operations warranted adjustment. A provider explained a problem related to the undesirable situation of leaving a patient sitting alone while the linkage coordinator traveled from a different clinic to reach the patient:

"I don't have capacity to sit in the room for 30 minutes while we wait for someone to come. Our nursing team doesn't have that capacity. So a lot of times for a substantial amount of that period, the patient is sitting alone in an office exam room, living with that diagnosis. And I always thought that that was not ideal. A lot can happen in your head in 30 minutes in a room alone." (FQHC KI05)

As a result of this type of observation, the FQHC responded by shifting the order of who would meet with the patient to fill in the linkage specialist's travel time and eventually added an in-house linkage person at this clinic.

(7) Strong communication methods and culture.

RAPID requires the coordination of numerous clinic staff to engage in multiple separate tasks simultaneously and quickly. Effective communication methods are essential. At the implementation level, 
effective communication of new and evolving protocols is necessary for everyone involved in the RAPID process to ensure understanding of their roles and how they function within the larger process. At the operations level communication is a key component of other essential elements, like flexibility, adaptability, and patient-centered care. The extent to which a RAPID visit can be tailored to a patient's specific needs and activated within variable clinic conditions is shaped in large part by the quality and efficiency of the communication between team members and across service areas.

Each site formalized RAPID communications differently. The Innovator Site and the FQHC employed a single point of contact for incoming RAPID cases (one used a pager system, the other used a RAPID phone staffed by linkage coordinators). The Testing Site operated within a compact space and when a client was diagnosed with HIV, the test counselor would give verbal notice to the lead clinician or speak directly to a nurse practitioner. Given the size of the facility and the close proximity of providers and staff to one another, it was not surprising to identify a pattern across interviewees illustrating that close collaboration and open communication among all providers and staff were hallmarks of the Testing Site clinic culture and appeared to be embedded within every clinical process.

Key informants also provided examples of communication breakdown at their site, further highlighting by contrast the importance of this element. At the Testing Site, fractured communication in the beginning across service areas troubled implementation roll-out:

"[Some of the] team actually wasn't even aware that we were trying to launch [RAPID], because they weren't really present [on-site]. They were primarily at the [other] site. And I think there was just bad communication happening at that time." (Test KI11)

At the FQHC, one informant described how the persistent ineffective communication of protocol changes in one area not only troubled RAPID operation, but presented a real danger to the sustainability of the program through damage to staff acceptability:

"They change things, and then they really don't give us directions on the next steps. They changed [to a new pharmacy software] and then didn't really train us on how to [use it]. ... It's little things like that that kind of happen frequently, with other examples. So, let's see. They changed ADAP on us. They just tell us things at the very, very, very last minute, and like the transparency is just like - by the time it gets to us, like we're already with a patient. And then it's just like, okay. And then you just have to figure it out as you go..." (FQHC KIO1)

This informant went on to attribute staff turnover to these "little things." Thus, determining effective communication strategies or course correcting when it was clear that communication was ineffective was critical for successful RAPID implementation.

\section{Challenges to Implementation with Implications for Sustainability}

Notable in these interviews was the duality of successful RAPID implementation along with stories of addressing challenges. While some informants insisted that RAPID was "easy," they also told stories of 
having to deal with transitional tension.

"For me as a clinician, [RAPID ART] was very easy, and it was a wonderful thing that we were able to offer it. So, for me, it was easy, logical, organic." (Test KI05)

"I think as time goes on, it's easier to manage and kind of predict, but in the beginning, that was a pretty tough transition." (FQHC KIO3)

Tensions that arose in interviews related to the transitions associated with moving from pre- to postRAPID programming, provider strain and burnout, the intensity of increased patient volume, and the stress of modifying RAPID processes to address changes over time. We offer these findings as a set of common challenges that may be more easily managed if they are expected.

\section{Transitional tension.}

Even for staff who were eager to be part of providing RAPID services, the transition to new processesincluding changes to schedules and workflow, and training and cross-training in new skills and dutieswas a challenge. Training in the RAPID protocol, medication access, and related benefits required time and energy, and was typically added on top of every-day duties. Cross-training, where staff who already possessed required competencies trained other team members, was an effective strategy to distribute RAPID duties more evenly across team members, but the process of cross-training could add trainer strain as well. An example is a PrEP navigator who leverages their medication access and eligibility assessment skills to occasionally assist RAPID clients. Training other staff in this skill set temporarily strains the PrEP navigator with a full load of PrEP navigation duties, yet cross-training on these essential competencies is the only way to eventually offload some of the task responsibility.

In some cases, transitional challenges were experienced as pain points that would eventually resolve (i.e., staffing or space reconfigurations), though not in all cases. Some informants reported instances of transition-related turnover, where occasionally a staff member would not be a good fit for the new competencies required by RAPID processes, or a change to staffing schedules was not acceptable to a person in an impacted role and consequently, they sought work outside of the organization.

The acute transition stage of implementation/iteration was typically a tense time, though multiple participants reported that the difficulty was temporary and improved over time. Thus, interpersonal friction and individual frustration can be anticipated, is transitional, and not necessarily a sign that a RAPID program is not feasible or sustainable at a particular site.

"Right now it's painful with all these transition periods, as we build out staff and training the staff. But the idea is that we'l be able to see folks without kind of derailing the clinic flow as much as it currently is and whenever certain situations pop up. So, that definitely seems like it's painful for now, but we're able to get through it." (Test KI11)

Burnout/provider strain.

Page 17/23 
Data from interviews showed high levels of buy-in for RAPID and that the implementation process was sustainable up to a point, sustainability became problematic when staff and provider strain threatened to lead to burnout. Staff who did not feel sufficiently supported or were already overtaxed prior to RAPID implementation were at particular risk of burnout. One prescribing provider who took a lot of pride in their work and their connection with their clients talked about both appreciating the emotional authenticity and engagement of the RAPID interaction, but also wished that there was an opportunity, "even 15 minutes" to decompress, maybe take a walk around the block, before returning to seeing other patients. Another interviewee explained the strain of balancing the duties of engaging in a RAPID encounter against the work of caring for their existing client caseload.

"This is just the same-day start in general. Like when they implement these programs, they should talk to the people who are going to be doing it for more input, because these people are getting burnt out really quickly. ... they're just burnt out, like tired. And then not only are they doing same-day start, but they have a full caseload of 40 individuals that are newly positive that they have to retain and engage in care. So, it's not just a same-day start, and then that's it. Your job is not done. You have to maintain contact with these people, schedule their monthly or bimonthly medical appointments, meet them at their appointment, try to get them to come to support groups, be their sometimes sole supporter, make sure they have their medication, check in on them, see how they're doing, make sure they're taking their medication. If they're not taking their medication, see what the problem is. Like it's way more." (FQHC KIO1)

\section{Volume.}

The number of RAPID visits a site executes over a period of time, or RAPID volume, was reported as a high-impact factor in program sustainability. A primary constraint that clinics came up against was that of capacity: "how many [RAPID encounters] can you do?" Even the most seamless RAPID program is only seamless up to a point; a program designed to absorb the disruption and added work associated with a RAPID visit that is seeing one RAPID case a month is a fundamentally different program than one that is seeing one a week, or one a day.

"Originally I thought we were going to be doing 12 a year. .... So, that was my initial proposal for it. And then when all these people came in, I think we did like 65 last year. I'm like, that's more than 12." (Test K104)

"During the next year, 2017-2018, it became increasingly less manageable. The numbers were growing, our staff was not." (Test KIO3)

Both adopter sites struggled with an unanticipated increase in volume as their RAPID programs took root. The Testing Site began receiving increased referrals as other agencies learned of their success with RAPID, while program success led to overall growth at the FQHC, outpacing provider capacity:

"[The RAPID program we implemented] was just meant to be for our folks. And then what was happening is people were testing positive at other locations, and that location couldn't take care of them, so they 
were funneling them to us. You know, so we kind of became this referral center." (Test KI04)

"One of the difficult things we're now facing is that [our site] and same-day start is growing so rapidly that like just follow-up appointments are really difficult. So, in the beginning, you were supposed to have a follow-up appointment one to two weeks out from diagnosis. But now, it's like a month to a month and a half just because all of the providers are so booked, and a lot of them don't even have open panels [and] scheduling that initial follow-up is so difficult. And then if somebody misses that follow-up appointment, we're not seeing them for like three months after their initial diagnosis just because scheduling is so hard." (FQHC KIO3)

Needs may change over time.

Related to the need for adaptive capacity, the needs of a RAPID program will almost certainly evolve over time. A site may end up needing more staff, needing to change clinic flow, or needing more physical space. Additionally, future ways of immediately accessing ART medications may change. One site recognized as their program progressed that the mental health needs that arose for some of their patients as part of the diagnosis and disclosure experience would be best served by in-house mental health services. Another site recognized that RAPID as a program could better serve patients if some key staff worked four 10-hour shifts in a week rather than five 8-hour shifts. Some changes may arise from iterative quality improvement as a clinic seeks to optimize services and patient and provider experience, while some changing needs may be driven by conditions such as funding fluctuations or COVID-19 restrictions.

\section{Discussion}

RAPID implementation relies less on the topical application of new elements onto an existing clinic structure than it does on the coordination of a clinic's existing human resources and the cultivation of systems and processes that support unpredictable needs and patient-centered care. Practical application of the seven essential elements described above means: identifying where these elements currently exist within a practice; leveraging them when possible; strengthening them when necessary or developing them if they do not yet exist; and looking to these elements when challenges arise for potential solutions.

Reports of success and ease with the provision of RAPID services were attributed, either directly or indirectly, to strong communication, high adaptive capacity, and a commitment to patient-centered care, while many of the challenges relayed by informants reflected situations in which flexibility had reached a limit or communication had broken down. Our finding that these elements are the essential building blocks of a successful RAPID program should not be interpreted to mean that these elements were wholly present or executed perfectly at each site. Indeed, identifying instances where a weak or lacking component posed a threat to a program was as informative as identifying the inverse.

It is important to note that the operational structure of RAPID programs is shaped by the clinical setting in which they function, and therefore the essential elements identified herein will not apply equally to all 
programs. Some settings have onsite primary care, which reduces or may eliminate the role of linkage, compared to settings that have to link patients to external primary HIV care. Some settings receive newly diagnosed patients as referrals from outside their system while others do not-a factor that can affect the volume of RAPID patients. Evidence suggests that, in the United States, settings with existing HIV testing programs or strong referral partnerships between testing and clinic entities may be in a position to implement a RAPID program without adding significant resources. ${ }^{11,12}$ In locations with weak HIV testing and ART delivery infrastructure and limited human resources, implementation of RAPID programming may be less feasible.

The goal of this analysis was to identify essential elements of RAPID programs-components that would ideally be present for successful implementation of RAPID. These elements are similar to, but distinct from, facilitators and best practices. The 'implementation champion' was reported on as an essential element here, however moving forward it may prove to be a nice, but not necessary element. The literature suggest and our data support the importance of a champion in facilitating the successful implementation of a novel intervention; this may be especially important during the innovation and early adoption phases of diffusion of an innovation. Once an innovation becomes more mainstream, the role of the champion becomes less critical. Future RAPID implementers will need to determine whether their setting warrants identification or cultivation of a RAPID champion. Relatedly, champions often serve to build buy-in for an innovation. While we briefly described provider buy-in as a key component of the comfort and competence to prescribe RAPID ART, we take up this concept more fully in a forthcoming publication.

This study is not without limitations. The sites selected for analysis are well-resourced and already highly skilled in providing culturally competent care to people living with HIV. Our findings may be less transferable outside of those contexts. Our sample from the FQHC site was smaller relative to the other sites, and notably more potential participants declined to participate in that site than the others. This could have been because providers at this site were less experienced with RAPID services and may have felt they would not be able to meaningfully contribute to the study. Providers at this site may have been too busy to prioritize participation in a research study. Finally, the geographic difference and weaker ties between the research team and the prospective participants in the FQHC site may have been a further deterrent to participation.

Future research should provide insights into how the new paradigm of RAPID ART becomes normalized in a clinical setting. Such research should also be improved upon with the addition of a more granular, ground-level exploration of how individual providers approach the RAPID interaction with patients.

\section{Conclusion}

Based on extensive interviews with RAPID staff, we provide evidence that the RAPID model can be applied to a diverse range of clinical contexts with a higher degree of provider/staff acceptability than was previously known, and a synthesis of the essential elements necessary to do so. These essential 
elements are not inclusive of all facilitators identified in this study; rather they are the essential elements common across sites, without which the programs may not have been able to operate. These essential elements may be easily achieved in some clinical settings-such as those supported by the Ryan White HIV/AIDS Program-and difficult to achieve in other settings without, for example, a willing prescriber or a reliable payer source to cover the costs of medication, labs, and clinic visits. We are optimistic about future development of RAPID programming throughout the U.S. and hope future implementers of RAPID will take inspiration from the examples of the three successful programs outlined here to create a program consisting of some or all of these essential elements, and work towards developing the 'ingredients' that may not yet exist in their environments.

\section{Abbreviations}

ADAP AIDS drug assistance program; APN advanced practice nurse, ART antiretroviral therapy; CFIR Consolidated Framework for Implementation Research, DO doctor of osteopathic medicine, FQHC federally qualified health center, HE health educator, LTC linkage to care coordinator, MD medical doctor, MSW master of social work, NP nurse practitioner, PA physician's assistant, RN registered nurse

\section{Declarations}

Ethics approval and consent to participate: Approval for the study (\#18-25758) was obtained by the University of California, San Francisco Institutional Review Board. Verbal informed consent was obtained from the participants after explaining fully the purpose of the study and their rights as participants. The consent procedure was approved by the University of California, San Francisco Institutional Review Board. Confidentiality was ensured and participation in the study was voluntary. All methods were carried out in accordance with relevant guidelines and regulations.

Consent for publication: Not applicable.

Availability of data and materials: The dataset used and/or analysed during the current study are available from the corresponding author on reasonable request.

Competing interests: The authors declare that they have no competing interests.

Funding: This study was supported by the California HIV/AIDS Research Program and Gilead Sciences.

Authors' contributions: Contributed substantially to the design of the work (K.A.C, K.A.K), acquisition of the data (K.A.K., L.M., L.V., J.B., P.C.C., J.S.), analysis of the data (K.A.K., L.M., N.L., K.A.C.). K.A.K and L.M. drafted the manuscript. All authors revised the manuscript for important intellectual content and approved the final version.

Acknowledgements: The authors wish to thank all of the participants who took part in this study. 


\section{References}

1. Cohen MS, Chen YQ, McCauley M, et al. Antiretroviral Therapy for the Prevention of HIV-1 Transmission. The New England journal of medicine. 2016;375(9):830-839. doi:10.1056/NEJMoa1600693

2. Cohen MS, Chen YQ, McCauley M, Gamble T, Hosseinipour MC et al. Prevention of HIV-1 infection with early antiretroviral therapy. N Engl J Med. 2011;365:493-505.

3. Farel CE, Dennis AM. Why Everyone (Almost) with HIV Needs to Be on Treatment. Infectious Disease Clinics of North America. 2019;33(3). doi:10.1016/j.idc.2019.05.002

4. Rosen S, Maskew M, Fox MP, et al. Rapid art initiation reduces loss between HIV testing and treatment: The Rapit trial. Top Antivir Med. 2015;23(1).

5. Zhao Y, McGoogan JM, Wu Z. The Benefits of Immediate ART. Journal of the International Association of Providers of AIDS Care. 2019;18. doi:10.1177/2325958219831714

6. Danel. A Trial of Early Antiretrovirals and Isoniazid Preventive Therapy in Africa. New England Journal of Medicine. 2015;373(9):808-822. doi:10.1056/NEJMoa1507198

7. INSIGHT Start Study Group. Initiation of Antiretroviral Therapy in Early Asymptomatic HIV Infection. New England Journal of Medicine. 2015;373(9). doi:10.1056/NEJMoa1506816

8. Henn A, Flateau C, Gallien S. Primary HIV Infection: Clinical Presentation, Testing, and Treatment. Current Infectious Disease Reports. 2017;19(10). doi:10.1007/s11908-017-0588-3

9. Halperin J, Conner K, Butler I, et al. A Care Continuum of Immediate ART for Newly Diagnosed Patients and Patients Presenting Later to Care at a Federally Qualified Health Center in New Orleans. Open Forum Infectious Diseases. 2019;6(4). doi:10.1093/ofid/ofz161

10. Ford N, Migone C, Calmy A, et al. Benefits and risks of rapid initiation of antiretroviral therapy. AIDS. 2018;32(1):17-23. doi:10.1097/QAD.0000000000001671

11. Gomillia CES, Backus K v., Brock JB, Melvin SC, Parham JJ, Mena LA. Rapid Antiretroviral Therapy (ART) Initiation at a Community-Based Clinic in Jackson, MS. AIDS Research and Therapy. 2020;17(1). doi:10.1186/s12981-020-00319-7

12. McNulty M, Schmitt J, Friedman E, et al. Implementing Rapid Initiation of Antiretroviral Therapy for Acute HIV Infection Within a Routine Testing and Linkage to Care Program in Chicago. Journal of the International Association of Providers of AIDS Care. 2020;19. doi:10.1177/2325958220939754

13. Colasanti J, Sumitani J, Christina Mehta C, et al. Implementation of a rapid entry program decreases time to viral suppression among vulnerable persons living with HIV in the southern United States. Open Forum Infectious Diseases. 2018;5(6). doi:10.1093/ofid/ofy104

14. Coffey S, Bacchetti P, Sachdev D, et al. RAPID antiretroviral therapy: High virologic suppression rates with immediate antiretroviral therapy initiation in a vulnerable urban clinic population. AIDS. 2019;33(5):825-832. doi:10.1097/QAD.0000000000002124

15. Pilcher CD, Ospina-Norvell C, Dasgupta A, et al. The Effect of Same-Day Observed Initiation of Antiretroviral Therapy on HIV Viral Load and Treatment Outcomes in a US Public Health Setting. 
Journal of Acquired Immune Deficiency Syndromes. 2017;74(1).

doi:10.1097/QAI.0000000000001134

16. Boyd MA, Boffito M, Castagna A, Estrada V. Rapid initiation of antiretroviral therapy at HIV diagnosis: definition, process, knowledge gaps. HIV Medicine. 2019;20(S1):3-11. doi:10.1111/hiv.12708

17. Mgbako O, Sobieszczyk ME, Olender S, et al. Immediate antiretroviral therapy: The need for a health equity approach. International Journal of Environmental Research and Public Health. 2020;17(19):110. doi:10.3390/ijerph17197345

18. Damschroder LJ, Aron DC, Keith RE, Kirsh SR, Alexander JA, Lowery JC. Fostering implementation of health services research findings into practice: a consolidated framework for advancing implementation science. Implementation Science. 2009;4(1):50. doi:10.1186/1748-5908-4-50

19. SocioCultural Research Consultants L. Dedoose Version 8.3.35. Published online 2018.

20. Birt L, Scott S, Cavers D, Campbell C, Walter F. Member Checking: A Tool to Enhance Trustworthiness or Merely a Nod to Validation? Qualitative Health Research. Published online 2016. doi:10.1177/1049732316654870

21. Shortell SM, Marsteller JA, Lin M, et al. The Role of Perceived Team Effectiveness in Improving Chronic Illness Care. Medical Care. 2004;42(11). doi:10.1097/00005650-200411000-00002

22. Miech EJ, Rattray NA, Flanagan ME, Damschroder L, Schmid AA, Damush TM. Inside help: An integrative review of champions in healthcare-related implementation. SAGE Open Medicine. 2018;6. doi:10.1177/2050312118773261

23. Slaunwhite JM SSFMSRLC. Increasing vaccination rates among health care workers using unit "champions" as a motivator. . Can J Infect Control . 2009;Fall 24(3):159-164.

24. Weiser J, Beer L, West BT, Duke CC, Gremel GW, Skarbinski J. Qualifications, Demographics, Satisfaction, and Future Capacity of the HIV Care Provider Workforce in the United States, 20132014. Clinical Infectious Diseases. 2016;63(7). doi:10.1093/cid/ciw442

25. Petterson SM, Liaw WR, Phillips RL, Rabin DL, Meyers DS, Bazemore AW. Projecting US Primary Care Physician Workforce Needs: 2010-2025. The Annals of Family Medicine. 2012;10(6):503. doi:10.1370/afm.1431 